\title{
There are Infinitely Many Fibonacci Primes
}

Abstract- We invent a novel algorithm and solve the Fibonacci prime conjecture by an interaction between proof and algorithm. From the entire set of natural numbers successively deleting the residue class 0 mod a prime, we retain this prime and possibly delete another one prime retained, then we invent a recursive sieve method, a modulo algorithm on finite sets of natural numbers, for indices of Fibonacci primes. The sifting process mechanically yields a sequence of sets of natural numbers, which converges to the index set of all Fibonacci primes. The corresponding cardinal sequence is strictly increasing. The algorithm reveals a structure of particular order topology of the index set of all Fibonacci primes, then we readily prove that the index set of all Fibonacci primes is an infinite set based on the existing theory of the structure. Some mysteries of primes are hidden in second order arithmetics.

Keywords and phrases: Fibonacci prime conjecture, recursive sieve method, order topology, limit of a sequence of sets.

GJSFR-F Classification: MSC 2010: 11N35, 11N32, 11U09, 11Y16, 11B37, 11B50

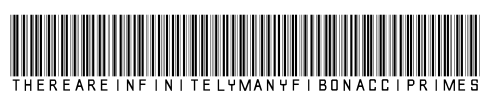

Strictly as per the compliance and regulations of:

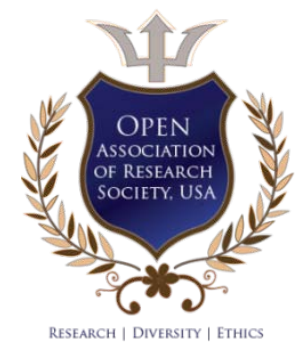

(C) 2020. Fengsui Liu. This is a research/review paper, distributed under the terms of the Creative Commons AttributionNoncommercial 3.0 Unported License http://creativecommons.org/licenses/by-nc/3.0/), permitting all non commercial use, distribution, and reproduction in any medium, provided the original work is properly cited. 

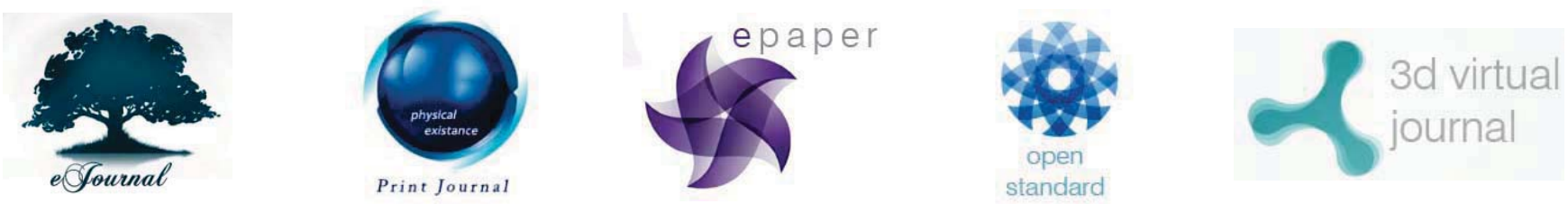

\title{
There are Infinitely Many Fibonacci
}

\author{
Primes
}

Fengsui Liu

Abstract-We invent a novel algorithm and solve the Fibonacci prime conjecture by an interaction between proof and algorithm. From the entire set of natural numbers successively deleting the residue class 0 mod a prime, we retain this prime and possibly delete another one prime retained, then we invent a recursive sieve method, a modulo algorithm on finite sets of natural numbers, for indices of Fibonacci primes. The sifting process mechanically yields a sequence of sets of natural numbers, which converges to the index set of all Fibonacci primes. The corresponding cardinal sequence is strictly increasing. The algorithm reveals a structure of particular order topology of the index set of all Fibonacci primes, then we readily prove that the index set of all Fibonacci primes is an infinite set based on the existing theory of the structure. Some mysteries of primes are hidden in second order arithmetics.

Keywords and phrases: Fibonacci prime conjecture, recursive sieve method, order topology, limit of a sequence of sets.

\section{INTRODUCTION}

Fibonacci numbers $F_{x}$ are defined by the recursive formula

$$
\begin{aligned}
F_{1} & =1, \\
F_{2} & =1, \\
F_{x+1} & =F_{x}+F_{x-1} .
\end{aligned}
$$

The first few Fibonacci numbers are:

$1,1,2,3,5,8,13,21,34,55,89,144,233,377,610,987,1597,2584$, 4181, 6765, 10946, 17711, 28657, 46368, ... (OEIS A005478)

A Fibonacci prime is a Fibonacci number that is prime.

The first few Fibonacci primes are:

2, 3, 5, 13, 89, 233, 1597, 28657, 514229, 433494437, 2971215073, ... (OEIS A005478).

The first few indices $x$, which give Fibonacci primes are:

3, 4, 5, 7, 11, 13, 17, 23, 29, 43,47, 83, 131, 137, 359, 431, 433, 449, 509, $569,571,2971,4723,5387, \ldots$ (OEIS A001605). 
The study of Fibonacci primes has a long history. At present, Fibonacci primes with thousands of digits have been found. For example, 33-th Fibonacci prime is $F_{81839}$, with 17103 digits. We do not repeat those stories.

From the aspect of primality, like the Mersenne numbers [7], we have an open problem and a conjecture.

There are infinitely many Fibonacci composites with prime indices.

There are infinitely many Fibonacci primes[12][1].

In another paper, we had discussed the open problem [7].

In this paper, we prove the Fibonacci prime conjecture by a recursive sieve method or algorithm.

In order to be self-contained we repeat some contents in papers [7].

\section{A Sifting Process for Primes}

For expressing a recursive sieve method by well-formed formulas, we extend both operations addition and multiplication,$+ \times$ into finite sets of natural numbers. We introduce several definitions and notations.

We use small letters $a, x, t$ to denote natural numbers and capital letters $A, X, T$ sets of natural numbers except for $F_{x}$.

For arbitrary both finite sets of natural numbers $A, B$ we write

$$
\begin{aligned}
& A=\left\langle a_{1}, a_{2}, \ldots, a_{i}, \ldots, a_{n}\right\rangle, a_{1}<a_{2}<\cdots<a_{i}<\cdots<a_{n}, \\
& B=\left\langle b_{1}, b_{2}, \ldots, b_{j}, \ldots, b_{m}\right\rangle, b_{1}<b_{2}<\cdots<b_{j}<\cdots<b_{m} .
\end{aligned}
$$

We define

$$
\begin{gathered}
A+B=\left\langle a_{1}+b_{1}, a_{2}+b_{1}, \ldots, a_{i}+b_{j} \ldots, a_{n-1}+b_{m}, a_{n}+b_{m}\right\rangle, \\
A B=\left\langle a_{1} b_{1}, a_{2} b_{1}, \ldots, a_{i} b_{j} \ldots, a_{n-1} b_{m}, a_{n} b_{m}\right\rangle .
\end{gathered}
$$

For example:

$$
\begin{gathered}
\langle 1,5\rangle+\langle 0,6,12,18,24\rangle=\langle 1,5,7,11,13,17,19,23,25,29\rangle \\
\langle 6\rangle\langle 0,1,2,3,4\rangle=\langle 0,6,12,18,24\rangle .
\end{gathered}
$$

For the empty set $\emptyset$ we define $\emptyset+B=\emptyset$ and $\emptyset B=\emptyset$.

We write $A \backslash B$ for the set difference of $A$ and $B$.

Let

$$
X \equiv A=\left\langle a_{1}, a_{2}, \ldots, a_{i}, \ldots, a_{n}\right\rangle \bmod a,
$$

be several residue classes $\bmod a$.

We define the solution of the system of congruences

$$
\begin{aligned}
& X \equiv A=\left\langle a_{1}, a_{2}, \ldots, a_{i}, \ldots, a_{n}\right\rangle \bmod a \\
& X \equiv B=\left\langle b_{1}, b_{2}, \ldots, b_{j}, \ldots, b_{m}\right\rangle \bmod b
\end{aligned}
$$


to be

$$
X \equiv D=\left\langle d_{11}, d_{21}, \ldots, d_{i j}, \ldots, d_{n-1 m}, d_{n m}\right\rangle \bmod a b,
$$

where $x \equiv d_{i j} \bmod a b$ is the solution of the system of congruences

$$
\begin{aligned}
& x \equiv a_{i} \bmod a, \\
& x \equiv b_{j} \bmod b .
\end{aligned}
$$

If $\operatorname{gcd}(a, b)=1$, the solution $X \equiv D \bmod a b$ is computable and unique by the Chinese remainder theorem.

For example, $X \equiv D=\langle 5,25\rangle \bmod 30$ is the solution of the system of congruences

$$
\begin{aligned}
& X \equiv\langle 1,5\rangle \bmod 6 \\
& X \equiv\langle 0\rangle \bmod 5 .
\end{aligned}
$$

We known that the residue class $a_{i} \bmod a$ is the set of natural numbers $\left\{x: x \equiv a_{i} \bmod a\right\}$, several residue classes $A \bmod a$ is the union of several sets. Thus we may write the relation $x \in A \bmod a$ and set operation $B \cup(A$ $\bmod a)$.

Except extending,$+ \times$ into finite sets of natural numbers, we continue the traditional interpretation of the formal language $0,1,+, \times, \in$. The reader who is familiar with model theory may know, we have found a new model or structure of the second order arithmetic by a two-sorted logic

$$
\langle P(N), N,+, \times, 0,1, \in\rangle,
$$

and we have found a formal system

$$
\langle P(N), N,+, \times, 0,1, \in\rangle \models P A \cup Z F,
$$

where $N$ is the set of all natural numbers, and $P(N)$ is the power set of $N$; $P A$ is the Peano theory and $Z F$ is the set theory.

We denote this model by $P(N)$.

In the traditional first order arithmetics $N$, we have computed out 33 Fibonacci primes, but the numerical evidence does not provide theoretically information about infinitude.

In the second order arithmetics $P(N)$, we may construct a recursive function on sets of natural numbers by arithmetical operations,$+ \times$ and set-theoretical operations $\cup, \cap, \backslash$, its inputs and outputs are sets of natural numbers, it expresses our intuitable idea: we delete all indices of nonFibonacci primes and retain all indices of Fibonacci primes. Then we obtain a sequence of sets of natural numbers $\left(T_{i}^{\prime}\right)$, which converges to the set $T_{e}$ of all indices of Fibonacci primes. We reveal an exotic structure of the set $T_{e}$ 
in second order arithmetics. The existing theory of those structures, order topology, allows us to prove the conjecture.

First we repeat the recursively sifting process for primes.

Let $p_{i}$ be the $i$-th prime, $p_{0}=2$. Let

$$
m_{i+1}=\prod_{0}^{i} p_{j} .
$$

From the entire set of natural numbers we successively delete the residue class $0 \bmod p_{i}$, i.e., the set of all numbers $x$ such that the least prime factor of $x$ is $p_{i}$. We leave the residue class $T_{i+1} \bmod m_{i+1}$. Then the left residue class $T_{i+1} \bmod m_{i+1}$ is the set of all numbers $x$ such that $x$ does not contain any prime $p_{j} \leq p_{i}$ as a factor $\operatorname{gcd}\left(x, m_{i+1}\right)=1$.

Let $T_{i+1}$ be the set of least nonnegative representatives of the residue class $T_{i+1} \bmod m_{i+1}$, the reduced residue system $\bmod m_{i+1}$. We design a primitive recursive formula for the set $T_{i+1}$ and the prime $p_{i+1}$. This formula expresses a recursive sieve method for primes.

$$
\begin{aligned}
T_{1} & =\langle 1\rangle, \\
p_{1} & =3, \\
T_{i+1} & =\left(T_{i}+\left\langle m_{i}\right\rangle\left\langle 0,1,2, \ldots, p_{i}-1\right\rangle\right) \backslash\left\langle p_{i}\right\rangle T_{i}, \\
p_{i+1} & =g\left(T_{i+1}\right),
\end{aligned}
$$

where $X \equiv\left\langle p_{i}\right\rangle T_{i} \bmod m_{i+1}$ is the solution of the system of congruences

$$
\begin{aligned}
& X \equiv T_{i} \bmod m_{i}, \\
& X \equiv\langle 0\rangle \bmod p_{i},
\end{aligned}
$$

and $g(T)$ is a projective function

$$
g(T)=g\left(\left\langle t_{1}, t_{2}, \ldots, t_{n}\right\rangle\right)=t_{2} .
$$

The cardinality of the set $T_{i+1}$ is

$$
\left|T_{i+1}\right|=\prod_{0}^{i}\left(p_{j}-1\right) .
$$

For example:

$$
\begin{aligned}
& T_{1}=\langle 1\rangle, \\
& p_{1}=3, \\
& T_{2}=(\langle 1\rangle+\langle 2\rangle\langle 0,1,2\rangle) \backslash\langle 3\rangle=\langle 1,5\rangle, \\
& p_{2}=5, \\
& \left.T_{3}=\langle 1,5\rangle+\langle 6\rangle\langle 0,1,2,3,4\rangle\right) \backslash\langle 5,25\rangle=\langle 1,7,11,13,17,19,23,29\rangle . \\
& p_{3}=7 .
\end{aligned}
$$


It is easy to prove this primitive recursive formula by mathematical induction.

Look through a list of primes, the sequence of primes seems chaotic and random. It offers no clues as to how to determine the next number.

Randomness and chaos are anathema to the mathematician [10].

Despite over two thousand years one pursues an order, pattern, structure, and finds a formula that generates all primes exactly and without exception, our endeavour is in vain.

In the second arithmetics the prime formula 2.1 reveals the recursive structure of each primes. Now primes do not appear randomly. They are computed one after the other by,$+ \times$. They are governed by a recursive rule. Recursion opens new theoretical windows onto our understanding of the primes. It seems this is a prime conspiracy.

Gauss prime number theorem $\pi(x) \approx x / \ln x$ is an explicitly approximation for the recursive formula $\pi\left(p_{i}\right)=i$. The prime number theorem provides a heuristic model. Due to parity problem, we ought to reflect whether or not the heuristic model describes the sequence of primes enough accurately.

The prime formula is a reformulation of Eratosthenes sieve method.

In contrast with Eratosthenes sieve, which does not automatically yield theoretical information, the recursive sieve method itself mechanically yields a constructive proof that there are infinitely many primes[7].

We can not directly extend this constructive proof to Fibonacci primes.

Below we will refine and modify formula 2.1 to invent a recursive sieve for Fibonacci primes. A recursive formula on the sets of naturel numbers reveals a secret of the patterns of Fibonacci primes.

\section{iil. A Recursive Sieve for Fibonacci Primes}

Based on the recursive sieve method for primes, formula (2.1), we successively delete all numbers $x$ such that $x$ contains the least prime factor $p_{i}$, we delete all composites together with the prime $p_{i}$. The sifting condition or 'sieve' is

$$
x \equiv 0 \bmod p_{i} \wedge p_{i} \leq x .
$$

We refine the sifting condition to be

$$
x \equiv 0 \bmod p_{i} \wedge p_{i}<x .
$$

According to this new sifting condition or 'sieve', we successively delete the set $C_{i}$ of all numbers $x$ such that $x$ is composite with the least prime 
factor $p_{i}$,

$$
C_{i}=\left\{x: x \in X \equiv T_{i} \bmod m_{i} \wedge x \equiv 0 \bmod p_{i} \wedge p_{i}<x\right\}
$$

but save the prime $p_{i}$ as a survivor.

We delete all sets $C_{j}$ of composites with $0 \leq j<i$ from the entire set $N$ of natural numbers and leave the sifted set

$$
L_{i}=N \backslash \bigcup_{0}^{i-1} C_{j} .
$$

The end-sifted set, the set of all primes $T_{e}$, is

$$
T_{e}=N \backslash \bigcup_{0}^{\infty} C_{i} .
$$

Let $A_{i}$ be the set of all survivors less than $p_{i}$,

$$
A_{i}=\left\langle 2,3,5,7, \ldots, p_{i-1}\right\rangle .
$$

From the recursive formula (2.1), we deduce that the sifted set $L_{i}$ is the union of the set $A_{i}$ of survivors and the residue class $T_{i} \bmod m_{i}$,

$$
L_{i}=A_{i} \bigcup\left(T_{i} \bmod m_{i}\right)
$$

We slightly modify the above sifting process for primes to invent a recursive sieve for indices of Fibonacci primes.

We know that the Fibonacci sequence is a divisibility sequence. In fact, the Fibonacci sequence satisfies the stronger divisibility property[13]

$$
\operatorname{gcd}\left(F_{m}, F_{n}\right)=F_{\operatorname{gcd}(m, n)}
$$

In other words, for $n \geq 3, F_{n}$ divides $F_{m}$ iff $n$ divides $m$. It follows that except for the case $n=4, F_{4}=3$, every Fibonacci prime have a prime index, but not every prime is the index of a Fibonacci prime.

We discuss indices of Fibonacci primes and their infinitude in the set of indices of all Fibonacci numbers.

If we only consider Fibonacci numbers as factors of Fibonacci numbers within the Fibonacci divisibility system, then the set of all primes $T_{e}$ is the set of indices of all Fibonacci primes except for $F_{2}=1, F_{4}=3$. But some Fibonacci numbers with prime index contain normal prime factors that are not Fibonacci numbers. For example $F_{19}=4181=37 \times 113, F_{31}=$ $1346269=557 \times 2417$.

We must remove every prime index $q$ such that $F_{q}$ is a Fibonacci composite with a normal prime factor.

One uses the rank of a prime $p$ to discuss prime factors of Fibonacci composites with prime index. 
Definition: The rank of a prime $p$, denoted $\alpha(p)$, is the least positive integer $k$ such that $p \mid F_{k}$.

The first few ranks of primes are:

$3,4,5,8,10,7,9,18,24,14,30,19,20,44,16,27,58,15,68,70,37$, $78,84,11,49,50,104,36,27,19,128,130,69,46,37,50,79,164,168,87$, 178, 90, 190, 97, 99, 22, 42, 224, 228, 114, 13, 238, 120, 250, 129, 88, 67, $270,139,28,284,147,44,310, \ldots \ldots$ (sequence A001602 in the OEIS).

We exhibit some simple facts about normal prime factors of Fibonacci composites with prime index.

(1) A prime number divides at most one Fibonacci number with prime index due to the stronger divisibility property.

(2) If $q>4$ is a prime, then every prime $p$ that divides $F_{q}$ is congruent to $1 \bmod 4$ [8].

(3) Let $p>5$ be a prime, if $p \equiv \pm 1 \bmod 10$, then $\alpha(p) \mid p-1$; if $p \equiv$ $\pm 3 \bmod 10$, then $\alpha(p) \mid p+1[5]$. We have $\alpha(p) \leq \frac{p+1}{2}$.

(4) if $p>7$ is a prime such that $p \equiv 2,4 \bmod 5$, and $2 p-1$ is also prime, then $2 p-1 \mid F_{p}[14]$.

It follows that we only need to modify the set $A_{i}$ of survivors for each prime $p_{i}$ to obtain a new set $A_{i+1}$ of survivors, such that if $q \in A_{i+1}$ then $F_{q}$ contains neither normal prime $p \leq p_{i}$ nor Fibonacci prime $F_{p} \leq F_{p_{i}}$ as a factor except itself.

Given any prime $p_{i}>5$, suppose that we have a modified set $A_{i}$, then we obtain the next set $A_{i+1}$ by the following rules.

If the prime $p_{i}$ is congruent to $1 \bmod 4$, and if there is an odd prime $q \leq \frac{p_{i}+1}{2}$ in the set $A_{i}$ such that

$$
p_{i}<F_{q} \wedge \alpha\left(p_{i}\right)=q,
$$

then $F_{q}$ is a Fibonacci composite, which contains the least normal prime factor $p_{i}$. We add the prime $p_{i}$ into the set $A_{i}$ and remove the prime $q$ from the set $A_{i} \cup\left\langle p_{i}\right\rangle$ to obtain the set $A_{i+1}$.

$$
A_{i+1}=\left(A_{i} \cup\left\langle p_{i}\right\rangle\right) \backslash\langle q\rangle
$$

If there is no such a prime $q$, then for every number $x$ in the sifted set $L_{i}$ the Fibonacci number $F_{x}$ does not contain the normal prime $p_{i}$ as a factor. We add the prime $p_{i}$ into the set $A_{i}$

$$
A_{i+1}=A_{i} \cup\left\langle p_{i}\right\rangle \text {. }
$$


Now for every number $x$ in the sifted set $L_{i+1}$, the Fibonacci number $F_{x}$ does not contain Fibonacci number $M_{q}, q \leq p_{i}$ as a factor, and does not contain the normal prime $p_{j} \leq p_{i}$ as a factor except itself.

After modification the first few set $A_{i}$ are

$$
\begin{aligned}
A_{5} & =\langle 3,4,5,7.11\rangle \\
A_{8} & =\langle 3,4,5,7.11,13,17,19\rangle \\
A_{11} & =\langle 3,4,5,7.11,13,17,23,29,31,37\rangle, \\
A_{12} & =\langle 3,4,5,7.11,13,17,23,29,31,37,41\rangle, \\
A_{13} & =\langle 3,4,5,7.11,13,17,23,29,31,37,41,43\rangle, \\
A_{14} & =\langle 3,4,5,7.11,13,17,23,29,31,37,41,43,47\rangle .
\end{aligned}
$$

The sifting condition formula (3.1) is converted into

$$
\left(x \equiv 0 \bmod p_{i} \wedge p_{i}<x\right) \vee\left(F_{x} \equiv 0 \bmod p_{i} \wedge p_{i}<F_{x}\right) .
$$

According to this sifting condition or 'sieve', we successively delete the set $C_{i}$ of all numbers $x$, such that $F_{x}$ is composite with the least factor $F_{p_{i}}$, or $F_{x}$ is composite with the least normal prime factor $p_{i}>5$,

$$
\begin{array}{r}
C_{i}=\left\{x: x \in\left(A_{i} \cup\left(X \equiv T_{i} \bmod m_{i}\right)\right) \wedge\right. \\
\vee\left(F_{x} \equiv 0 \bmod p_{i} \wedge p_{i}<F_{x}\right) \\
\left.\left.\vee\left(x \equiv 0 \bmod p_{i} \wedge p_{i}<x\right)\right)\right\},
\end{array}
$$

but remain the survivor $x$ if $p_{i}=x$ or $p_{i}=F_{x}$.

We delete all sets $C_{j}$ with $0 \leq j<i$ from the set $N$ of all indices of Fibonacci numbers and leave a sifted set

$$
L_{i}=N \backslash \bigcup_{0}^{i-1} C_{j}
$$

The sifted set $L_{i}$ is descending

$$
L_{1} \supset L_{2} \supset \cdots \supset L_{i} \supset \cdots \cdots \text {. }
$$

The end-sifted set, the set $T_{e}$ of all indices of Fibonacci primes, is

$$
T_{e}=N \backslash \bigcup_{0}^{\infty} C_{i} .
$$

We easily verify that the primes $3,4,5,7.11,13,17,23,29,43,47$ all are indices of Fibonacci primes by the above algorithm.

The set $A_{i}$ of survivors is a set of indices of Fibonacci primes or probable primes, the candidates.

Obviously, we have

$$
\left|A_{i}\right| \leq\left|A_{i+1}\right|
$$


From the recursive formula (2.1), we deduce that the sifted set $L_{i}$ is the union of the set $A_{i}$ of survivors and the residue class $T_{i} \bmod m_{i}$.

$$
L_{i}=A_{i} \bigcup\left(T_{i} \bmod m_{i}\right)
$$

We intercept the initial segment $T_{i}^{\prime}$ from the sifted set $L_{i}$, which is the union of the set $A_{i}$ of survivors and the set $T_{i}$ of least nonnegative representatives, then we obtain a new recursive formula

$$
T_{i}^{\prime}=A_{i} \bigcup T_{i}
$$

Except remaining all survivors $x$ less than $p_{i}$ in the initial segment $T_{i}^{\prime}$, both sets $T_{i}^{\prime}$ and $T_{i}$ are the same.

For example

$$
\begin{aligned}
A_{3} & =\langle 3,4,5\rangle . \\
T_{3}^{\prime} & =\langle 3,4,5\rangle \cup\langle 1,7,11,13,17,19,23,29\rangle \\
& =\langle 3,4,5,1,7,11,13,17,19,23,29\rangle .
\end{aligned}
$$

Let $\left|A_{i}\right|$ be the number of survivors less than $p_{i}$. Then the number of elements of the initial segment $T_{i}^{\prime}$ is

$$
\left|T_{i}^{\prime}\right|=\left|A_{i}\right|+\left|T_{i}\right|
$$

From formula (2.2) we deduce that the corresponding cardinal sequence $\left(\left|T_{i}^{\prime}\right|\right)$ is strictly increasing

$$
\left|T_{i}^{\prime}\right|<\left|T_{i+1}^{\prime}\right|
$$

Based on cardinal arithmetics we have

$$
\lim T_{i}^{\prime}=\bigcup T_{i}^{\prime}=\aleph_{0}
$$

Based on order topology we have

$$
\lim \left|T_{i}^{\prime}\right|=\aleph_{0}
$$

Next section we will prove that the set of all indices of Fibonacci primes is an infinite set.

\section{The Infinitude of Fibonacci Primes}

We call an index of Fibonacci prime a F-index.

Let $A_{i}^{\prime}$ be the subset of F-indices in the initial segment $T_{i}^{\prime}$,

$$
A_{i}^{\prime}=\left\{x \in T_{i}^{\prime}: x \text { is a F-index }\right\} .
$$


For example:

$$
\begin{aligned}
& A_{2}^{\prime}=\langle 3,4,5\rangle \\
& A_{3}^{\prime}=\langle 3,4,5,7.11,13,17,23,29\rangle \\
& A_{4}^{\prime}=\langle 3,4,5,7,11,13,17,23,29,43,47,83,131,137\rangle .
\end{aligned}
$$

We consider the properties of both sequences of sets $\left(T_{i}^{\prime}\right)$ and $\left(A_{i}^{\prime}\right)$ to prove the conjecture.

Theorem 4.1. The sequence of the initial segments $\left(T_{i}^{\prime}\right)$ and the sequence of its subsets $\left(A_{i}^{\prime}\right)$ of F-indices both converge to the set of all F-indices $T_{e}$.

First from set theory [2], next from order topology [6] we prove this theorem.

Proof. For the convenience of the reader, we quote a definition of the settheoretic limit of a sequence of sets [2].

Let $\left(F_{n}\right)$ be a sequence of sets, we define $\lim \sup _{n=\infty} F_{n}$ and $\lim \inf _{n=\infty} F_{n}$ as follows

$$
\begin{aligned}
& \limsup _{n=\infty} F_{n}=\bigcap_{n=0}^{\infty} \bigcup_{i=0}^{\infty} F_{n+i}, \\
& \liminf _{n=\infty} F_{n}=\bigcup_{n=0}^{\infty} \bigcap_{i=0}^{\infty} F_{n+i} .
\end{aligned}
$$

It is easy to check that $\lim \sup _{n=\infty} F_{n}$ is the set of those elements $x$ which belongs to $F_{n}$ for infinitely many $n$. Analogously, $x$ belongs to $\lim \inf _{n=\infty} F_{n}$ if and only if it belongs to $F_{n}$ for almost all $n$, that is it belongs to all but a finite number of the $F_{n}$.

If

$$
\limsup _{n=\infty} F_{n}=\liminf _{n=\infty} F_{n}
$$

we say that the sequence of sets $\left(F_{n}\right)$ converges to the limit

$$
\lim F_{n}=\limsup _{n=\infty} F_{n}=\liminf _{n=\infty} F_{n} .
$$

We know that the sequence of sifted sets $\left(L_{i}\right)$ is descending

$$
L_{1} \supset L_{2} \supset \cdots \supset L_{i} \supset \cdots \cdots .
$$

According to the definition of the set-theoretic limit of a sequence of sets, we obtain that the sequence of sifted sets $\left(L_{i}\right)$ converges to the set $T_{e}$

$$
\lim L_{i}=\bigcap L_{i}=T_{e}
$$

The sequence of subsets $\left(A_{i}^{\prime}\right)$ of F-indices is ascending

$$
A_{1}^{\prime} \subset A_{2}^{\prime} \subset \cdots \subset A_{i}^{\prime} \subset \cdots \cdots
$$


we obtain that the sequence of subsets $\left(A_{i}^{\prime}\right)$ converges to the set $T_{e}$,

$$
\lim A_{i}^{\prime}=\bigcup A_{i}^{\prime}=T_{e}
$$

The initial segment $T_{i}^{\prime}$ locates between two sets $A_{i}^{\prime}$ and $L_{i}$

$$
A_{i}^{\prime} \subset T_{i}^{\prime} \subset L_{i}
$$

It is easy to prove that the sequence of the initial segments $\left(T_{i}^{\prime}\right)$ converges to the set $T_{e}$

$$
\lim T_{i}^{\prime}=T_{e}
$$

According to set theory, we have proved that both sequences of sets $\left(T_{i}^{\prime}\right)$ and $\left(A_{i}^{\prime}\right)$ converge to the set of all F-indices $T_{e}$.

$$
\lim T_{i}^{\prime}=\lim A_{i}^{\prime}=T_{e}
$$

In general, for any sequence of finite sets $\left(G_{i}\right)$, if $G_{i}$ locates between two sets $A_{i}^{\prime}$ and $L_{i}$,

$$
A_{i}^{\prime} \subset G_{i} \subset L_{i}
$$

then we have

$$
\begin{gathered}
\limsup G_{i} \subset \lim L_{i}, \\
\liminf G_{i} \supset \lim A_{i}^{\prime} .
\end{gathered}
$$

Thus

$$
\lim G_{i}=T_{e},
$$

Even $T_{e}=\emptyset$ the limit of set theory is valid too.

We can not use analytic techniques for limits of set theory so that we try to endow them with an order topology, and prove that both sequences of sets $\left(T_{i}^{\prime}\right)$ and $\left(A_{i}^{\prime}\right)$ converge to the set of all F-indices $T_{e}$.

We quote J.R.Munkres's definition of the order topology [4][6].

Let $X$ be a set with a linear order relation; assume $X$ has more one element. Let $\mathbb{B}$ be the collection of all sets of the following types:

(1) All open intervals $(a, b)$ in $X$.

(2) All intervals of the form $\left[a_{0}, b\right)$, where $a_{0}$ is the smallest element (if any) in $X$.

(3) All intervals of the form $\left[a, b_{0}\right)$, where $b_{0}$ is the largest element (if any) in $X$.

The collection $\mathbb{B}$ is a base of a topology on $X$, which is called the order topology.

The empty or singleton is not a linear order $<$ set. There is no order topology on the empty set or sets with a single element. For example a 
constant function $f(n)=0$ has a $\operatorname{limit} \lim f(n)=0$ in real analysis, but it has no limit in order topology, as $n \rightarrow \infty$.

The recursively sifting process formula (3.5) produces both sequences of sets together with the set-theoretic limit point $T_{e}$.

$$
\begin{gathered}
\mathbf{X}_{\mathbf{1}}: \quad T_{1}^{\prime}, T_{2}^{\prime}, \ldots, T_{i}^{\prime}, \ldots \ldots ; T_{e} \\
\mathbf{X}_{\mathbf{2}}: \quad A_{1}^{\prime}, A_{2}^{\prime}, \ldots, A_{i}^{\prime}, \ldots \ldots ; T_{e}
\end{gathered}
$$

We further consider the structures of sets $\mathbf{X}_{\mathbf{1}}$ and $\mathbf{X}_{\mathbf{2}}$ using the recursively sifting process (3.5) as an order relation

$$
\begin{aligned}
& i<j \rightarrow T_{i}^{\prime}<T_{j}^{\prime}, \forall i\left(T_{i}^{\prime}<T_{e}\right), \\
& i<j \rightarrow A_{i}^{\prime}<A_{j}^{\prime}, \forall i\left(A_{i}^{\prime}<T_{e}\right) .
\end{aligned}
$$

The set $\mathbf{X}_{\mathbf{1}}$ has no repeated term. It is a well ordered set with the order type $\omega+1$ using the recursively sifting process (3.5) as an order relation. Thus the set $\mathbf{X}_{\mathbf{1}}$ may be endowed an order topology.

In general, the set $\mathbf{X}_{\mathbf{2}}$ may have no repeated term, or may have some repeated terms, or may be a set with a single element $\mathbf{X}_{\mathbf{2}}=\{\emptyset\}$.

We have computed out some patterns of the first few F-indices. The set $\mathbf{X}_{\mathbf{2}}$ contains more than one element, may be endowed with an order topology. using the recursively sifting process (3.5) as an order relation.

Obviously, for every neighborhood $\left(c, T_{e}\right]$ of $T_{e}$, there is a natural number $i_{0}$, for all $i>i_{0}$, we have $T_{i}^{\prime} \in\left(c, T_{e}\right]$ and $A_{i}^{\prime} \in\left(c, T_{e}\right]$, thus both sequences of sets $\left(T_{i}^{\prime}\right)$ and $\left(A_{i}^{\prime}\right)$ converge to the set of all F-indices $T_{e}$.

$$
\begin{aligned}
\lim A_{i}^{\prime} & =T_{e} \\
\lim T_{i}^{\prime} & =T_{e} .
\end{aligned}
$$

According to the order topology, we have again proved that both sequences of sets $\left(T_{i}^{\prime}\right)$ and $\left(A_{i}^{\prime}\right)$ converge to the set of all F-indices $T_{e}$. We also have

$$
\lim T_{i}^{\prime}=\lim A_{i}^{\prime}
$$

If $T_{e}=\emptyset$, the set $\mathbf{X}_{\mathbf{2}}=\{\emptyset\}$ only has a single element, which has no order topology. In this case formula (4.2) is not valid and we prove nothing by the order topology.

Theorem (4.1) reveals a particular order topological structure of the set of all F-indices built into the sequences of sets. Now we easily prove that the cardinality of the set of all F-indices is infinite.

Theorem 4.2. The set of all F-indices is an infinite set. 
We give two proofs.

Proof. A. We consider the cardinalities $\left|T_{i}^{\prime}\right|$ and $\left|A_{i}^{\prime}\right|$ of sets on two sides of the equality (4.2), and the order topological limits of cardinal sequences $\left(\left|T_{i}^{\prime}\right|\right)$ and $\left(\left|A_{i}^{\prime}\right|\right)$ with the usual order relation $\leq$, as the sets $T_{i}^{\prime}$ and $A_{i}^{\prime}$ both tend to $T_{e}$.

From general topology, we know that if the limits of both cardinal sequences $\left(\left|T_{i}^{\prime}\right|\right)$ and $\left(\left|A_{i}^{\prime}\right|\right)$ on two sides of the equality (4.2) exist, then both limits are equal; if $\lim \left|A_{i}^{\prime}\right|$ does not exist, then the condition for the existence of the limit $\lim \left|T_{i}^{\prime}\right|$ is not sufficient [3].

For F-indices, the set $T_{e}$ is nonempty $T_{e} \neq \emptyset$, the formula (4.2) is valid, obviously, the order topological limits $\lim \left|A_{i}^{\prime}\right|$ and $\lim \left|T_{i}^{\prime}\right|$ on two sides of the equality (4.2) exist, thus both limits are equal

$$
\lim \left|A_{i}^{\prime}\right|=\lim \left|T_{i}^{\prime}\right|
$$

From formula (3.7) $\lim \left|T_{i}^{\prime}\right|=\aleph_{0}$ we have

$$
\lim \left|A_{i}^{\prime}\right|=\aleph_{0} .
$$

Usually, let $\pi(n)$ be the counting function, the number of F-indices less than $n$. Normal sieve theory is unable to provide non-trivial lower bounds of $\pi(n)$ due to the parity problem. Let $n$ be a natural number. Then the number sequence $\left(m_{i}\right)$ is a subsequence of the number sequence $(n)$, we obtain

$$
\lim \pi(n)=\lim \pi\left(m_{i}\right)
$$

By formula (4.1), the $A_{i}^{\prime}$ is the set of all F-indices less than $m_{i}$, and the $\left|A_{i}^{\prime}\right|$ is the number of all F-indices less than $m_{i}$, thus $\pi\left(m_{i}\right)=\left|A_{i}^{\prime}\right|$. We have

$$
\lim \pi\left(m_{i}\right)=\lim \left|A_{i}^{\prime}\right|
$$

From formula (4.3) we prove

$$
\lim \pi(n)=\aleph_{0} .
$$

Next, we give another proof by the continuity of the cardinal function.

Proof. B. Let $f: \mathbf{X} \rightarrow \mathbf{Y}$ be the cardinal function from the order topological space $\mathbf{X}$ to the order topological space $\mathbf{Y}$ such that $f(T)=|T|$.

$$
\begin{gathered}
\mathbf{X}: \quad T_{1}^{\prime}, T_{2}^{\prime}, \ldots, T_{i}^{\prime}, \ldots \ldots ; T_{e} \\
\mathbf{Y}:\left|T_{1}^{\prime}\right|,\left|T_{2}^{\prime}\right|, \ldots,\left|T_{i}^{\prime}\right|, \ldots \ldots ; \aleph_{0} .
\end{gathered}
$$

It is easy to check that for every open set $\left[\left|T_{1}^{\prime}\right|,|d|\right),(|c|,|d|),\left(|c|, \aleph_{0}\right]$ in $\mathbf{Y}$ the preimage $\left[T_{1}^{\prime}, d\right),(c, d),\left(c, T_{e}\right]$ is also an open set in $\mathbf{X}$. So that the 
cardinal function $|T|$ is continuous at $T_{e}$ with respect to the above particular order topology.

Both order topological spaces are first countable, hence the cardinal function $|T|$ is sequentially continuous. By a usual topological theorem [6] (Theorem 21.3, p130), the cardinal function $|T|$ preserves limits

$$
\left|\lim T_{i}^{\prime}\right|=\lim \left|T_{i}^{\prime}\right|
$$

Order topological spaces are Hausdorff spaces. In Hausdorff spaces the limit point of the sequence of sets $\left(T_{i}^{\prime}\right)$ and the limit point of cardinal sequence $\left(\left|T_{i}^{\prime}\right|\right)$ are unique.

We have proved theorem 4.1, $\lim T_{i}^{\prime}=T_{e}$, and formulas $(3.7), \lim \left|T_{i}^{\prime}\right|=$ $\aleph_{0}$. Substitute, we obtain that the set of all F-indices an infinite set,

$$
\left|T_{e}\right|=\aleph_{0}
$$

Without any estimation or statistical data, without the Riemann hypothesis, with the recursive sieve, we well understand the recursive structure, set theoretic structure and order topological structure of the set of all F-indices on sequences of sets. The theory of those structures allows us obtain an elementary proof of the conjecture.

We prove that the set of indices of all Fibonacci primes is a infinite set. In other words we have proved the theorem

Theorem 4.3. There are infinitely many Fibonacci primes.

\section{Discussion}

In general, we can not prove that the cardinal function on sequences of sets is continuous. There is a counterexample, the Ross-Littwood paradox [9] [11].

For example: consider the limit of the sequence of sets, which have no pattern

$$
T_{i}=\langle i+1, i+2, \ldots, 10 i\rangle
$$

From set theory we know $\lim T_{i}=T_{e}=\emptyset$, thus $\left|T_{e}\right|=0$. But we also have $\lim \left|T_{i}\right|=\infty$ from real analysis. If the cardinal function is continuous, then there is a contradiction in real analysis, the empty has an infinite cardinality. In this case, one can only get up the continuity and says that there is no relation between the cardinality of the end sifted set $\left|T_{e}\right|=0$ and the $\operatorname{limit} \lim \left|T_{i}\right|=\infty$. 
By the recursive sieve method, we have revealed that the set $T_{e}$ of all F-indices has the structure of a particular order topology

$$
\lim T_{i}^{\prime}=T_{e}
$$

So that we consider the conjecture in the particular order topological space, which is generated naturally by the recursively sifting process (3.5), rather than in real analysis, a metric space.

We consider all sequences of finite sets $\left(G_{i}\right)$, such that $A_{i}^{\prime} \subset G_{i} \subset L_{i}$, and they converge to the end sifted set $T_{e}$ from set theory

$$
\lim G_{i}=T_{e}
$$

We try to endow all set-theoretical convergence $\lim G_{i}=T_{e}$ with an order topology using the recursively sifting process (3.5) as an order relation, then construct a particular order topological space $\mathbf{G}$.

Here we must be careful about the existence of order topological limits.

First according to the definition, there exists no order topology on the empty set or sets with a single element[4] [6].

Next, we quote topologist L.D. Kudryavtsev's 'The existence of limits of a function' for $\lim \left|G_{i}\right|$.

If the space $\mathbf{X}$ satisfies the first axiom of countability at the point $T_{e}$ and the space $\mathbf{Y}$ is Hausdorff, then for the existence of the limit $\lim \left|G_{i}\right|$ of the cardinal function $\left|G_{i}\right|$, it is necessary and sufficient that for any sequence $\left(G_{i}\right)$, such that $\lim G_{i}=T_{e}$, the $\operatorname{limit} \lim \left|G_{i}\right|$ exists. If this condition holds, the limit $\lim \left|G_{i}\right|$ does not depend on the choice of the sequence $\left(G_{i}\right)$, and the common value of these limits is the limit of $\left(\left|G_{i}\right|\right)$ at $T_{e}$ [3].

If the end sifted set is empty $T_{e}=\emptyset$. Since the sequence of subsets $\left(A_{i}^{\prime}\right)$ is ascending, it converges to the set $T_{e}$ based on the recursively sifting process (3.5), from set theory we know that the set of sets of patterns $\mathbf{X}_{\mathbf{2}}: A_{1}^{\prime}, A_{2}^{\prime}, \ldots, A_{i}^{\prime}, \ldots \ldots, T_{e}$. only have a single element $\emptyset$ and the cardinal set $\mathbf{Y}_{\mathbf{2}}:\left|A_{1}^{\prime}\right|,\left|A_{2}^{\prime}\right|, \ldots,\left|A_{i}^{\prime}\right|, \ldots \ldots,\left|T_{e}\right|$. only have a single element 0 , both sets have no order topology, both sequences $\left(A_{i}^{\prime}\right),\left(\left|A_{i}^{\prime}\right|\right)$ have no limit of order topology.

Note, we consider the set sequences, the empty $\emptyset$ is as an element. If we find out at least one prime pattern, then the set sequence $\left(A_{i}^{\prime}\right)$ has more one element.

Only if the end sifted set is empty $T_{e}=\emptyset$, the $\operatorname{limits} \lim A_{i}^{\prime}$ and $\lim \left|A_{i}^{\prime}\right|$ have no existence. The existence of all other $\operatorname{limits} \lim \left|G_{i}\right|$ is not sufficient from the above general topology. Thus at the point $T_{e}=\emptyset$ there is no 
"continuous" or "non-continuous". There is no contradiction. In this case, one needs no order topology.

If the end sifted set is not empty $T_{e} \neq \emptyset$, since the inclusion relation $G_{i} \supset A_{i}^{\prime}$, the sequence $\left(A_{i}^{\prime}\right)$ has more one element, every sequence $\left(G_{i}\right)$ has more one element, every limit of set theory $\lim G_{i}$ may be endowed with an order topology. Every $\lim G_{i}$ has existence; every $\lim \left|G_{i}\right|$ has existence. The condition of existence of $\lim \left|T_{i}^{\prime}\right|, \lim T_{i}^{\prime}$ and $\lim \left|A_{i}^{\prime}\right|, \lim A_{i}^{\prime}$ is sufficient. Thus our proof of theorem 3.1 and 3.2 is correct.

In the formal system $P(N)$ we deal with the Ross-Littwood paradox and find out a proof of the Fibonacci prime conjecture in the particular order topological space.

The Ross-Littwood paradox shows that the restricted definition for order topology, assume $X$ has more one element, is necessary.

In fact, we consider the set of various prime patterns $T_{e}$, in advance, we have known at least one prime pattern, and in advance, we have known that the end sifted set is not empty $T_{e} \neq \emptyset$.

By the same paradigm, we may prove another prime conjecture, including the twin prime conjecture.

\section{ACKNOWLedgments}

Thank for Qian Liu in Hug Chinese for her support.

\section{References Références Referencias}

1. Chris Caldwell, The Top Twenty: Fibonacci Number from the Prime Pages. Retrieved 2017-03-03.

2. K. Kuratowsky and A. Mostowsky, set theory, With an introduction to descriptive set theory, North-Holland Publishing com.(1976).

3. L. D. Kudryavtsev, Encyclopaedia of Mathematics, limit, [Online] Available: http://eom.springer.de/l/1058820.htm.

4. Jesper M. Møller, General Topology web, math.ku,dk (2005)20.

5. Marc Renault, The Fibonacci Sequence Modulo m. http:// webspace.ship.edu/ msrenault/fibonacci/fib.htm.

6. J. R. Munkres, Topology, (2rd ed.), Prentice Hall, Upper Saddle River, (2000), 84.

7. Fengsui Liu, On the Sophie Germain Prime Conjecture WSEAS Transactions on mathematics, 1012 (2011), 421-430.

Fengsui Liu, Which polynomials represent infinitely many primes. Global Journal of Pure and Applied Mathematics, Volume 14, Number 1 (2018), 161-180.

Fengsui Liu, There are infinitely many Mersnne composite numbers with prime exponents. Advances in Pure Mathematics, 2018, 8, 686-698.

Fengsui Liu, There are infinitely many Fibonacci composite numbers with prime subscripts, Research and Communications in Mathematics and Mathematical Sciences, Vol 10,2, 2018, 123-140. 
Fengsui Liu, There are infinitely many Mersnne primes, London jurnal of reseach in sience: natural and formal. Volume 20 - Issue 3 - Compilation 1.0. 23-42.

8. Lemmermeyer, Franz (2000), Reciprocity Laws, New York: Springer, ISBN 3-540-66957-4.

9. J. E. Littlewood, [1953], Littlewood's Miscellany, (ed. Bela Bollobas), Cambridge University Press, Cambridge, (1986), p.26.

10. M. D. Sautoy, The Music of the Primes, HarperCollins Publishers, (2003), p.6.

11. S. Ross, A First Course in Probability, (3rd ed.), New York and London, Macmillan, (1988).

12. Steven Vajda. Fibonacci and Lucas Numbers, and the Golden Section: Theory and Applications. Dover Books on Mathematics.

13. Ribenboim, Paulo, My Numbers, My Friends, Springer-Verlag, (2000).

14. V. Drobot, On primes in the Fibonacci sequence. Fibonacci Quarterly, 1998, 38 (1):71-72. 